discussions in medical notes. Information obtained from this audit will help guide development and implementation of new, combined DNACPR/TEPP forms in relation to new nationwide schemes.

\section{WORKING IN PARTNERSHIP IN THE COMMUNITY: OUR EXPERIENCE AND OUTCOMES OF THE LONDON AMBULANCE SERVICE CALLS TO ST LUKE'S HOSPICE (HARROW AND BRENT) SINGLE POINT OF ACCESS SERVICE}

R Carrigan, R Anderson, I Tipping, J Archer, C Daniels. St. Luke's Hospice (Harrow \& Brent), Kenton, Harrow

\subsection{6/bmjspcare-2018-ASPabstracts. 18}

In May 2015, St Luke's Hospice launched a Single Point of Access (SPA) telephone and triage service. The aim of this service was to provide a coordinated 24/7 telephone service to provide specialist advice and assistance to patients in the last year of life. The SPA is also available to health and social care professionals, including paramedics from London Ambulance Service (LAS). The SPA service also provides access to a rapid response team to provide direct care to patients in need between 7 am-to-11 pm.

All telephone calls made between the SPA and LAS paramedics were logged onto a clinical database (iCare). By searching for the appropriate call code, relevant calls from the first 20 months of the SPA service were identified and analysed.

There were 130 telephone calls; 90 were regarding patients known to the SPA (67 individual patients), and 40 were about patients previously unknown to the service. Taking into account multiple calls for the same event, 74 separate clinical events were analysed. The most common outcome of a call from LAS was to arrange a call/visit from a GP (usually out of hours) or by a district nurse (DN) (in or out of hours) $28 \%$. A further $12 \%$ of the calls required a visit from the hospice rapid response team, and only $11 \%$ of patients were escorted to hospital.

Analysis of these calls highlights the importance of the SPA as a liaison service, with over $25 \%$ of calls resulting in callouts from community services (GP, DN, community palliative teams), and a further $12 \%$ requiring a call-out from the SPA rapid response team. We believe this is the first collection of data to show the impact of a palliative SPA service on the LAS, facilitating clinical decision-making, and improving confidence in leaving patients at home with community support.

\section{THE IMPACT OF AN INTEGRATED RENAL SUPPORTIVE CARE SERVICE ON SYMPTOM BURDEN, ADVANCED CARE PLANNING AND PLACE OF DEATH FOR PATIENTS WITH ADVANCED CHRONIC KIDNEY DISEASE MANAGED WITHOUT DIALYSIS}

CA Douglas, JM Sloan, M Witham, S Cathcart, L Stage, L Frame, ME Lafferty. NHS Tayside, University of Dundee

10.1136/bmjspcare-2018-ASPabstracts. 182

Background We redesigned a Renal Supportive Care (RSC) service in 2012 for patients with chronic kidney disease
(CKD) managed without dialysis. We audited the service to evaluate impact on symptoms and care pathways.

Methods The RSC service allows consultations over a wide geographical area and includes a RSC nurse, a Renal consultant and a Palliative Medicine consultant. The main focus is continuing care of CKD, symptom management and Advanced Care Planning.

A retrospective case-note audit was performed from April 2012 until October 2014 on all known patients who had chosen non-dialytic management. Data collected included demographics, RSC input, Palliative Care Outcome Score (POS), anticipatory care plan (ACP) information and mortality data.

Results 98 patients were managed with non-dialytic care during the audit period. 72\% (71/98) received RSC consultations. $72 \%(57 / 79)$ of patients who received a RSC consultation had a full assessment of symptoms using the POS with an improvement in total symptom burden from a median score of 11 to 8 , between the first and last consultation $(p=0.03)$.

For patients with RSC input, 79\% (56/71) had an ACP compared to $19 \%(5 / 27)$ without $(\mathrm{p}<0.001)$. Preferred Place of Care (PPC) was documented in 68\% (48/71) and 26\% (7/ $27)$ in each respective group $(\mathrm{p}<0.001)$. PPC was community in $100 \%$. DNA CPR was documented in $84 \%(57 / 68)$ of patients with RSC input and 47\% $(8 / 17)$ without $(\mathrm{p}=0.001)$.

During the audit, $62 \%(61 / 98)$ of patients died. 29\% (11/ 38 ) of patients with an ACP died in an acute hospital versus $43 \%(10 / 23)$ of those without $(p=0.25) .24 \%(8 / 34)$ of patients with documented PPC died in acute hospitals versus $48 \%(13 / 27)$ without documentation $(\mathrm{p}=0.04)$.

Conclusions The Renal Supportive Care team achieved symptom management and Advanced Care Planning for many patients with CKD managed without dialysis and may help achieve preferred place of care.

\section{A PROSPECTIVE AUDIT OF SYMPTOM BURDEN AND PALLIATIVE CARE REFERRAL AMONGST IN-PATIENTS WITH DECOMPENSATED LIVER DISEASE}

A Brown-Kerr, D Trolland, I Hay, S Barclay, F Finlay. Glasgow Royal Infirmary, Queen Elizabeth University Hospital Glasgow

10.1136/bmjspcare-2018-ASPabstracts. 183

Aim Previous studies have suggested an unmet symptom burden in patients with decompensated chronic liver disease (DCLD). The Bristol Prognosis Score (BPS) has been designed to identify patients with DCLD likely to be in the last year of their life, who may benefit from Specialist Palliative Care team input. We aimed to audit the prevalence of symptoms appropriate to trigger referal to the Hospital Specialist Palliative Care Team (HSPCT), (using the Integrated Palliative care Outcome Score (IPOS)), rates of recognition of these by physicians, and rates of referral to HPSCT according to BPS status. Methods A prospective audit of all inpatients with DCLD admitted over 3 months was conducted. Patients completed IPOS questionnaires. Demographic data were collated in order to calculate BPS. Case notes were reviewed following discharge to assess if significant symptoms (regarded as scoring $\%$ \% 2 on IPOS) were identified by the medical team. Referral rates to HSPCT were also recorded.

Results 40 patients with DCLD were included. 36 (90\%) scored \%o¥2 in at least one IPOS category. Most patients (20 $(55.6 \%))$ had only some of their significant symptoms 
recognised by medical staff. 27/40 (67.5\%) patients were BPS positive. Of these only $1(3.7 \%)$ was referred to HSPCT.

Conclusions This work demonstrates a significant symptom burden in patients with DCLD. This does not appear to be recognised by the medial team, meaning referral rates to HSPCT are low. Routine use of the IPOS in conjunction with BPS may aid identification and referral of patients to HSPCT, and help address this.

\section{OPIOID THERAPY IN CHRONIC CANCER PAIN - ARE WE DOING THE RIGHT THING?}

Hannah Clare, Lisa La Mola, Richard Berman. The Christie NHS Foundation Trust

\subsection{6/bmjspcare-2018-ASPabstracts. 184}

Background People are surviving cancer for longer and are undergoing more lines of treatments. Their survival may be complicated by chronic pain for which they can be on longterm opioids. The pitfalls of chronic opioid use are well documented in non-cancer pain. At The Christie, there are increasing referrals to the Supportive Care Team (SCT) for patients with uncontrolled pain, on high doses of opioids experiencing significant adverse effects, whilst being actively treated for cancer.

Aims Assess the response in pain levels, side effects and ability to remain on treatment following opioid reduction, and adoption of a more targeted approach to pain control.

Method A retrospective case note review was conducted. Those included had been referred with worsening chronic pain as the predominant symptom, and an opioid reduction within the initial management plan. Data was collected with an audit tool at the initial and subsequent visit.

Results Nine case notes were reviewed. The mean opioid reduction was 35\% (range 20\%-100\%). All patients reported a reduction in their self-rated pain score with $78 \%$ reporting at least an improvement of 2 points (out of 10). All those who felt constipated reported an improvement, and 83\% who reported drowsiness felt it had resolved. At $89 \%$ of clinic visits a non-opioid medication was either started or titrated. Breakthrough opioid doses were altered in all (one excluded as no documentation) with $50 \%$ having a dose reduction (average $37.25 \%$ ) and $62.5 \%$ starting trans-mucosal fentanyl. There were no delays in those on active treatment.

Conclusion Reducing overall opioid dose, treating breakthrough pain as a separate entity and optimising non-opioid medications improved both pain control and the adverse effects of opioids.

Early referral to the SCT can help optimise pain control, reduce side effects and allow patients to maintain their performance status to continue treatment.

\section{THE 'FORGOTTEN' GENERATION: QUALITY OF LIFE IN MEN LIVING WITH DUCHENNE MUSCULAR DYSTROPHY}

Sheonad Laidlaw, Emma Carduff. Marie Curie Care, University of Cardiff

10.1136/bmjspcare-2018-ASPabstracts. 185

Background Duchenne Muscular Dystrophy (DMD) is an $\mathrm{x}^{-}$ linked, ultra-rare neuromuscular condition affecting 1 in 3600-6000 live male births. Life expectancy is increasing thanks to targeted intervention and advancing medical treatment. Longer survival and advancing age means a greater dependence on family, friends and the wider community, and increasing social and health care costs. However, little is known about quality of life (QoL) in adults with DMD.

Aim To investigate and explore quality of life in men living with DMD in the West of Scotland.

Methods This was a qualitative interview study using the 'Schedule for the Evaluation of Individual Quality of life Direct Weighting' (SEIQoL-DW) tool, an interview based instrument which allows the assessment of elements that contribute to an individual's QoL. Men over the age of 25 and living in the West of Scotland were identified and recruited through the Scottish Muscle Network - a multidisciplinary clinical network. The interviews were transcribed and analysed thematically applying descriptive labels to the data.

Results Six men were recruited and interviewed. A number of themes were characterised as key to good QoL: strong personal relationships providing support and advocacy; meaningful connection with the world through hobbies and on-line communities; being in control of physical and mental well being, supported by knowledgeable and approachable health care professionals; continued independence through use of essential equipment both in and out of the adapted accessible home; and a positive, resilient attitude to life with a disability. Conclusions Key improvements could improve QoL in this 'forgotten' group of adults: upskilling and support for all care givers; lifelong input from physiotherapy; timely access to psychological support; improved access to respite facilities; better co-ordinated holistic multidisciplinary care; and proactive advance care planning.

\section{ENHANCED SUPPORTIVE CARE (ESC): RESULTS FROM THE NATIONAL CQUIN DATA (YEAR 1)}

Richard Berman, Lisa La Mola, Elizabeth Elliott, Nathan Hall, Jill Lockhart. NHS England, The Christie NHS Foundation Trust

\subsection{6/bmjspcare-2018-ASPabstracts.186}

Background The landscape of cancer care is changing rapidly. As more patients live longer with 'chronic cancer' and new treatments become increasingly available, there is a growing need for oncologists, palliative care specialists and acute physicians to contribute more to the development of expertise in managing problems associated with long-term cancer and cancer survival. ESC is a national cancer centre initiative which aims to implement early involvement of supportive care within oncology. The ultimate aim of ESC is to make available supportive care expertise for patients at any stage, including those with curable cancers.

Methods ESC is being delivered through a national cquin. This has encouraged additional investment allowing resource expansion required to deliver ESC. In most centres, development of ESC has been led by palliative care clinicians and incorporated within palliative care teams. In other centres, this is led by oncologists. Some 'rebranded' the name of their palliative care team to include 'supportive care' or 'symptom control'; whilst others have developed separate 'ESC teams' within their centre. In this 1st phase of ESC, referrals to supportive care are encouraged for patients who are within 6 weeks of a diagnosis of incurable cancer.

Results ESC is a 3-year national project (2016-2019). 23 cancer centres across England signed up to the initiative in 